\title{
A Novel Extracorporeal Cardiopulmonary Resuscitation Strategy Using a Hybrid Emergency Room for Patients With PEA
}

Shinichi ljuin ( $\square$ shinichiijuin821@yahoo.co.jp )

Hyogo Emergency Medical Center https://orcid.org/0000-0003-3385-1533

\section{Akihiko Inoue}

Hyogo Emergency Medical Center

\section{Satoshi Ishihara}

Hyogo Emergency Medical Center

\section{Masafumi Suga}

Hyogo Emergency Medical Center

\section{Takeshi Nishimura}

Hyogo Emergency Medical Center

Shota Kikuta

Hyogo Emergency Medical Center

\section{Haruki Nakayama}

Hyogo Emergency Medical Center

Nobuaki Igarashi

Japanese Red Cross Kobe Hospital

\section{Shigenari Matsuyama}

Hyogo Emergency Medical Center

Tomofumi Doi

Japanese Red Cross Kobe Hospital

\section{Shinichi Nakayama}

Hyogo Emergency Medical Center

\section{Research Article}

Keywords: Extracorporeal cardiopulmonary resuscitation, Hybrid emergency room, Pulseless electrical activity, Pulmonary embolism, Aortic disease, Intracranial haemorrhage

Posted Date: March 7th, 2022

DOI: https://doi.org/10.21203/rs.3.rs-1410483/v1 
License: (c) (i) This work is licensed under a Creative Commons Attribution 4.0 International License. Read Full License 


\section{Abstract}

Background: Whether extracorporeal cardiopulmonary resuscitation (ECPR) is indicated for pulseless electrical activity (PEA) remains unclear. Pulmonary embolism with PEA was a good candidate for ECPR; however, PEA sometimes include an aortic disease and intracranial haemorrhage, with extremely poor neurological outcomes, and could not be a candidate. We have induced an ECPR strategy using the hybrid emergency room (ER) to perform computed tomography (CT) before extracorporeal membrane oxygenation (ECMO) induction from January 2020. Therefore, this study aimed to evaluate the effectiveness of our ECPR strategy.

Methods: Medical records of patients who transferred to our hybrid emergency room (ER) and required ECPR for PEA between January 2020 and November 2021 were reviewed.

Results: Twelve consecutive patients (median age, 67 [range, 57-73] years) with PEA requiring ECPR were identified in our hybrid ER. Among them, nine were diagnosed through initial CT scan (intracranial haemorrhage, 3; cardiac tamponade due to aortic dissection, 3; aortic rupture, 2; and cardiac rupture, 1), and unnecessary ECMO was avoided. The remaining three patients underwent ECPR, and two of them survived with favourable neurological outcomes. All patients not indicated for ECPR were excluded before ECMO induction.

Conclusion: Our ECPR strategy using the hybrid ER may be useful for the exclusion of patients with PEA non-indication for ECPR and decision making.

\section{Background}

Extracorporeal cardiopulmonary resuscitation (ECPR) is a method used to stabilize hemodynamics and provide end-organ perfusion in case of inadequate conventional CPR and reversible cause of cardiac arrest (CA). A favourable neurological outcome is likely to be achieved by ECPR for CA patients with a shockable rhythm, which is ventricular fibrillation (VF) or pulseless ventricular tachycardia (pVT) on the initial electrocardiogram (ECG) $[1,2]$. Therefore, previous studies of ECPR have selected shockable rhythm as the initial rhythm. Otherwise, the indication of ECPR for pulseless electrical activity (PEA) remains unclear. The clinical challenge is how to identify patients with PEA.

Several studies have reported successful resuscitation inducing ECPR for patients with PEA [3-5]. Among CA patients with PEA, pulmonary embolism was a good indication for ECPR [6-9]; however, PEA sometimes includes aortic disease and intracranial haemorrhage $[10,11]$, which were extremely poor [1214], and could not be a candidate for ECPR. Therefore, these diseases should be distinguished before extracorporeal membrane oxygenation (ECMO) induction. However, they are difficult to diagnose without imaging evaluation.

The advent of interventional radiology-computed tomography (IVR-CT) has had a considerable effect on the management of trauma patients, enabling diagnosis and intervention without transferring the patient 
simultaneously. The hybrid emergency room (ER) was an ER equipped with IVR-CT [15]. The use of a hybrid ER reportedly decreases mortality in patients with severe trauma by reducing the time until wholebody computed tomography (CT) imaging and allowing early initiation of definitive therapy $[15,16]$. Furthermore, a hybrid ER has been reported to allow safer and rapid cannulation and ECMO initiation [9, 17] and to allow the excursion of patients not indicated for ECPR before ECMO induction.

We launched a novel ECPR strategy using a hybrid ER to perform CT before ECMO induction to exclude non-indication of ECPR for patients with out-of-hospital cardiac arrest (OHCA) patients from January 2020. Therefore, this study aimed to assess the effectiveness of our ECPR strategy.

\section{Methods}

\section{Study design and setting}

This retrospective descriptive study was performed at Hyogo Emergency Medical Center, a tertiary emergency medical center, in Japan. Patients are brought to our institution based on emergency medical services (EMS)'s judgements. The medical records of patients with OHCA who were identified from the database were reviewed, and their clinical features and relevant data were collected. This study was approved by Hyogo Emergency Medical Center ethical committee (approval number: 2020008). Written informed consent for using patients' data was obtained from their families at the time of admission by the attending physician.

A hybrid ER was installed at our ER in January 2017 and is equipped with a multi-slice IVR-CT system (Aquilion PRIME, TSX-303A; Canon Medical Systems Corp, Tochigi, Japan). Our hybrid ER has a carbonfiber fluoroscopic table with a movable C-arm combined with a sliding gantry CT scanner (Fig. 1), equipment that allows us to perform diagnostic and therapeutic procedures simultaneously in a single space. At our institution, all trauma patients are taken to the hybrid ER whenever available. Medical patients with unstable vital signs, indicated for ECPR who have PEA, or intracranial diseases such as brain infarction or haemorrhage are also taken to this room.

\section{Patients}

Patients with OHCA who were transferred to the hybrid ER at Hyogo Emergency Medical Center between January 2020 and November 2021 and required ECPR for PEA were included in this study. In our institution, ECPR is indicated for the following: patients aged 16-75 years, initial ECG rhythm was shockable regardless of witnessed arrest, initial ECG rhythm was PEA with witnessed arrest, and the time from CA to hospital arrival within $45 \mathrm{~min}$. ECPR was defined as implantation of VA-ECMO in patients with OHCA who did not show the return of spontaneous circulation on hospital arrival. Patients with VF/VT are directly transferred to the angiography room and immediately induced with ECMO. 


\section{Procedure}

Patients with PEA for the ECPR candidate were transferred to the hybrid ER. Mechanical CPR was continuously performed using an automatic chest compression device (ACCD) (CLOVER3000; KOHKEN Medical, Tokyo, Japan). Then, to shorten the time, head and chest CT scan is immediately carried out without scout images under mechanical CPR (Fig. 1). If CT findings did not detect intracranial haemorrhage, pericardial effusion or massive haemorrhage in the pleural space, venoarterial extracorporeal membrane oxygenation (VA-ECMO) is started. However, if these findings were found, conventional CPR was performed without ECMO induction, because the patient is strongly expected to have unfavourable neurological outcomes (Fig. 2). The implementation of ECPR was finally decided by the emergency physician. All ECMO cannulations were performed by the emergency physician and cardiologist. The cannulas were inserted into the femoral artery and vein under ultra-sonographic and fluoroscopic guidance. We used 17-Fr cannulas for the femoral artery and $21-\mathrm{Fr}$ for the femoral vein. As necessary, an additional 4-Fr catheter was inserted into the superficial femoral artery to prevent limb ischemia. The final position of the cannulas and catheters was confirmed by fluoroscopy.

\section{Data collection}

Data were retrieved from EMS personnel reports, and in-hospital data were retrieved from medical records. We collected data on age, sex, shock delivery from the arrival of EMS to the establishment of ECMO support, time from hospital arrival to the end of CT, the arrival-to-ECMO time (duration from hospital arrival to the establishment of ECMO support onset) and low-flow time (duration from CA or predicted CA to the establishment of ECMO support onset), CA etiology and survival and neurological outcomes at hospital discharge. Neurological outcomes were assessed with Glasgow-Pittsburg Cerebral Performance Category scale [18]. Continuous variables are presented as median (interquartile range $[\mathrm{IQR}])$.

\section{Results}

During the study period, a total of 271 patients with OHCA were transferred to our institute. Among them, 34 patients were ECPR candidates, and 22 of them were excluded from this study, because 21 patients had initial ECG rhythm with a shockable rhythm and one was induced ECMO before performing a CT scan due to the physician's decision. Finally, the remaining 12 patients were included in this study (Fig. 3).

The baseline characteristics and clinical course of these patients are shown in Table. 1. The median age of patients was 67 (IQR, 57-73) years and nine were male. The collapse-to-arrival time and time from arrival to the end of CT were $22(17-28) \mathrm{min}$ and 170 (160-188) seconds, respectively. Among them, nine patients were diagnosed by CT scan without a scout image (intracranial haemorrhage, 3 ; cardiac tamponade due to acute aortic dissection (AAD), 3; aortic rupture, 2; and cardiac rupture, 1) (Fig. 4). The remaining three patients underwent ECPR because they had no findings of intracranial haemorrhage, 
pericardial effusion and massive haemorrhage in the pleural space by CT scan without a scout image. The arrival-to-ECMO time was 12, 14 and $13 \mathrm{~min}$, and the low-flow time was 40, 33 and $21 \mathrm{~min}$, respectively (Case 3, 6 and 8). They were diagnosed with acute coronary syndrome due to occlusion of the left main trunk, aortic dissection complicated with right coronary artery malperfusion without pericardial effusion and pulmonary embolism. No complications related to ECMO induction were observed. Two (Case 6 and 8) of them survived with favourable neurological outcomes. In Case 6, as no findings of cardiac tamponade or rupture were observed on CT scan regardless of AAD, this patient had favourable outcomes. One patient (Case 3 ) died on day 12 due to low output syndrome caused by acute coronary syndrome. All patients not indicated for ECPR were excluded before ECMO induction, and unnecessary ECMO was avoided.

\section{Discussion}

In this study, we evaluated the efficacy of ECPR for patients with PEA in hybrid ER.

Twelve patients with PEA were transferred as ECPR candidates and were assessed whether ECPR can be indicated or not due to our strategy. Nine patients didn't undergo unnecessary ECMO induction, and two of three patients who underwent ECPR had favourable neurological outcomes.

Several studies have reported successful resuscitation inducing ECPR for patients with PEA $[3-5,19]$. Diek et al. reported that the survival rate of patients with PEA for ECPR was 23.8\% [20]. As shockable rhythm is a good candidate for ECPR [1, 2], non-shockable rhythms may also be a candidate for ECPR [21]. Patients with PEA with witness are more likely to have a fatal pulmonary embolism [6], and the favourable outcomes were expected inducing $\operatorname{ECMO}[7,8]$. Hence, it seems that CA due to pulmonary embolism can be expected to also have a favourable neurological outcome by inducing ECPR. Furthermore, some reports demonstrated the use of ECPR for accidental hypothermia with PEA [22, 23]. The disease can be diagnosed based on medical history and physical findings, and aggressive ECMO induction may lead to improve neurological outcomes.

Those with poor neurological outcomes of ECPR due to aortic disease and intracranial haemorrhage were not indicated for ECPR [12]. Although ECPR for intracranial haemorrhage has not been reported, CA due to this disease has been reported to have unfavourable neurological outcomes $[13,14]$. These patients could not be considered a candidate for ECPR. Therefore, whether ECPR is indicated or not before ECMO induction should be distinguished. However, in our study, one AAD patient without cardiac tamponade and rupture had a favourable neurological outcome (Case 6). Regardless of CA due to AAD, patients without cardiac tamponade and rupture, for example, coronary artery malperfusion may be a candidate for ECPR.

In conventional ECPR performed in the ER or angiography room, these diseases cannot be diagnosed before ECMO induction. A hybrid ER enables a diagnosis made on CT scan before ECMO induction and to simultaneously induced ECMO without relocating patient. Furthermore, the use of fluoroscopy may be advantageous in avoiding incorrect cannula placement and bleeding complications [17]. Therefore, our 
protocol is more useful compared to conventional ECPR. In our study, all patients with diseases not indicated for ECPR were excluded due to the initial CT scan and to avoid unnecessary ECMO in hybrid ER. Therefore, our strategy would contribute to the ECPR cost effectiveness.

This study has several limitations to this study. First, it is a retrospective, single center study. Second, the number of patients might be small, and the investigation was based on a case series targeting a relatively limited number of patients. Third, treatment decision-making depends on the physician.

\section{Conclusions}

Our ECPR strategy using hybrid ER may contribute to diagnosing patients not indicated for ECPR, such as those with intracranial haemorrhage and aortic disease with cardiac tamponade and rupture before ECMO induction, which may be useful for decision making of ECPR. However, information on hybrid ERs is limited in the literature; therefore, further research on their efficacy is needed.

\section{Abbreviations}

AAD: acute aortic dissection

ACCD: automatic chest compression device

CA: cardiac arrest

CT: computed tomography

ECG: electrocardiogram

ECMO: extracorporeal membrane oxygenation

ECPR: extracorporeal cardiopulmonary resuscitation

EMS: emergency medical services

Hybrid ER: Hybrid emergency room

IQR: interquartile range

IVR-CT: interventional radiology-computed tomography

OHCA: out-of-hospital cardiac arrest

pVT: pulseless ventricular tachycardia

PEA: pulseless electrical activity 
VA ECMO: venoarterial extracorporeal membrane oxygenation

VF: ventricular fibrillation

\section{Declarations}

\section{Ethics approval and consent to participate}

This study was approved by Hyogo Emergency Medical Center ethical committee (approval number: 2020008), which complied with the tenets of the Declaration of Helsinki. The requirement for patient consent was waived due to the retrospective nature of the study. Written informed consent for using patients' data was obtained from their families at the time of admission by the attending physician.

\section{Consent for publication}

Not applicable

\section{Availability of data and materials}

The dataset used and analyzed during the current study are available from the corresponding author on reasonable request.

\section{Competing interests}

The authors declare no conflicts of interest.

\section{Fundings}

No funding was received for this research from any source.

\section{Author's contributions}

S. ljuin was involved in main work, data collection, and manuscript writing. S. ljuin and Al designed and coordinated the study. S. Ishihara, MS, TN, SK, HN, NI, SM, TD and SN were involved in data collection and manuscript revision. Al and SN was involved in final revision. All authors read and approved the final manuscript.

\section{Acknowledgements:}

The authors are grateful to medical doctors of the Department of Cardiology, Japanese Red Cross Kobe Hospital.

\section{References}


1. Sakamoto T, Morimura N, Nagao K, Asai Y, Yokota H, Nara S, et al. Extracorporeal cardiopulmonary resuscitation versus conventional cardiopulmonary resuscitation in adults out-of-hospital cardiac arrest: a prospective observational study. Resuscitation 2014;85:762-8.

2. Yannopoulos D, Bartos J, Raveendran G, Walser E, Connett J, Murray TA, et al. Advanced reperfusion strategies for patients with out-of-hospital cardiac arrest and refractory ventricular fibrillation: a phase 2, single center, open-label, randomized controlled trial. Lancet 2020 ;396:1807-16.

3. Mehra C, Brady W. Pulseless electrical activity in cardiac arrest: electrocardiographic presentations and management considerations based on the electrocardiogram. Am J Emerg Med 2012;30:236-94.

4. Andrew E, Nehme Z, Lijovic M, Bernard S, Smith K. Outcomes following out-of- hospital cardiac arrest with an initial cardiac rhythm of asystole or pulseless electrical activity. Resuscitation 2014;85: 63339.

5. Huang HW, Chiu CC, Yen HH, Chen YL, Siao FY. Prolonged pulseless electrical activity: successful resuscitation using extracorporeal membrane oxygenation. Am J Emrg Med 2015;33: 474.e5-615.

6. Courtney DR, Sasser HC, Pincus CL, Pincus CL, Kline JA. Pulseless electrical activity with witnessed arrest as a predictor of sudden death from massive pulmonary embolism in outpatients. Resuscitation 2001;49:265-72.

7. Hashiba K, Okuda J, Maejima N, Iwahashi N, Tsukahara K, Tahara Y. Percutaneous cardiopulmonary support in pulmonary embolism with cardiac arrest. Resuscitation 2012;83:183-7.

8. Mandigers L, Scholten E, Rietdijk W JR, Uil CA, Thiel RJ, Rigter S, et al. Survival and neurological outcome with extracorporeal cardiopulmonary resuscitation for refractory cardiac arrest caused by massive pulmonary embolism: A two center observational study. Resuscitation 2019;136:8-13.

9. Miyazaki K, Hikone M, Kuwahara Y, Ishida T, Sugiyama K, Hamabe Y. Extracorporeal CPR for massive pulmonary embolism in a "hybrid emergency department". Am J Emerg Med 2019;37:2132-5.

10. Virkkunen I, Paasio L, Ryynanen S, Vuori A, Sajantila A, Yli-Hankala A, et al. Pulseless electrical activity and unsuccessful out-of-hospital resuscitation: What is the cause of death? Resuscitation 2008;77: 207-10.

11. Morris NA, Robinson D, Schmidt JM, Frey HP, Park S, Agarwal S, et al. Hunt-Hess 5 subarachnoid haemorrhage presenting with cardiac arrest is associated with larger volume bleeds. Resuscitation 2018;123:71-6.

12. Ohbe $\mathrm{H}$, Ogura $\mathrm{T}$, Matsui $\mathrm{H}$, Yasunaga $\mathrm{H}$. Extracorporeal cardiopulmonary resuscitation for acute aortic dissection during cardiac arrest: A nationwide etrospective observational study. Resuscitation 2020;156:237-43.

13. Toussaint LG $3^{\text {rd }}$, Friedman JA, Wijdicks EF, Piepgras DG, Pichelmann MA, Mclver JI, et al. Survival of cardiac arrest after aneurysmal subarachnoid hemorrhage. Neurosurgery 2005;57:25-31.

14. Meurer WJ, Walsh B, Vilke GM, Coyne CJ. Clinical guidelines for emergency department evaluation of subarachnoid hemorrhage. J Emerg Med 2016;50:696-701. 
15. Kinoshita T, Yamakawa K, Matsuda H, Yoshikawa Y, Wada D, Hamasaki T, et al. The survival benefit of a novel trauma workflow that includes immediate whole-body computed tomography, surgery and interventional radiology, all in one trauma resuscitation room $₫ A$ retrospective historical control study. Ann Surg 2019;269: 370-6

16. Wada D, Nakamori Y, Yamakawa K, Fujimi S. First clinical experience with IVR-CT system in the emergency room: Positive impact on trauma workflow. Scand J Trauma Resusc Emerg Med 2012;20:52.

17. Kashiura M, Sugiyama K, Tanabe T, Akashi A, Hamabe Y. Effect of ultrasonography and fluoroscopic guidance on the incidence of complications cannulation in extracorporeal cardiopulmonary resuscitation in out-of-hospital cardiac arrest: a retrospective observational study. BMC Anesthesiol 2017;17:4.

18. Cummins RO, Chamberlain DA, Abramson NS, Allen M, Baskett BJ, Becker L, et al. Recommended guidelines for uniform reporting of data from out-of-hospital cardiac arrest: the Utstein Style. A statement for health professionals from a task force of the American Heart Association, the European Resuscitation Council, the Heart and Stroke Foundation of Canada, and the Australian Resuscitation Council. Circulation 1991;84:960-75.

19. Iloha L, Lucie F, Hana S, Ondrej S, Ales L, Helena K, et al. Refractory ventricular arrhythmias alternating with pulseless electrical activity in a young woman rescued by extracorporeal cardiopulmonary resuscitation. Case Rep Med 2018;1-4.

20. Dirk P, Christoph EB. Is pulseless electrical activity a reason to refuse cardiopulmonary resuscitation with ECMO support? Am J Emerg Med. 2018;36: 637-40.

21. Tanimoto A, Sugiyama K, Tanabe M, et al. Out-of-hospital cardiac arrest patients with an initial nonshockable rhythm could be candidates for extracorporeal cardiopulmonary resuscitation: a retrospective study. Scand J Trauma Resusc Emerg Med. 2020;28:101

22. Sawamoto K, Bird SB, Katayama Y, Maekawa K, Uemura S, Tanno K, et al. Outcome from severe accidental hypothermia with cardiac arrest resuscitated with extracorporeal cardiopulmonary resuscitation. Am J Emerg Med 2014; 32: 320-4.

23. Pasquier M, Rousson V, Darocha T, et al. Hypothermia outcome predicted after extracorporeal life support for hypothermic cardiac arrest patients: An external validation of the HOPE score. Resuscitation 2019;139: 321-8.

\section{Table}

Table 1 PDF is available in the Supplemental Files section.

\section{Figures}




\section{Figure 1.}

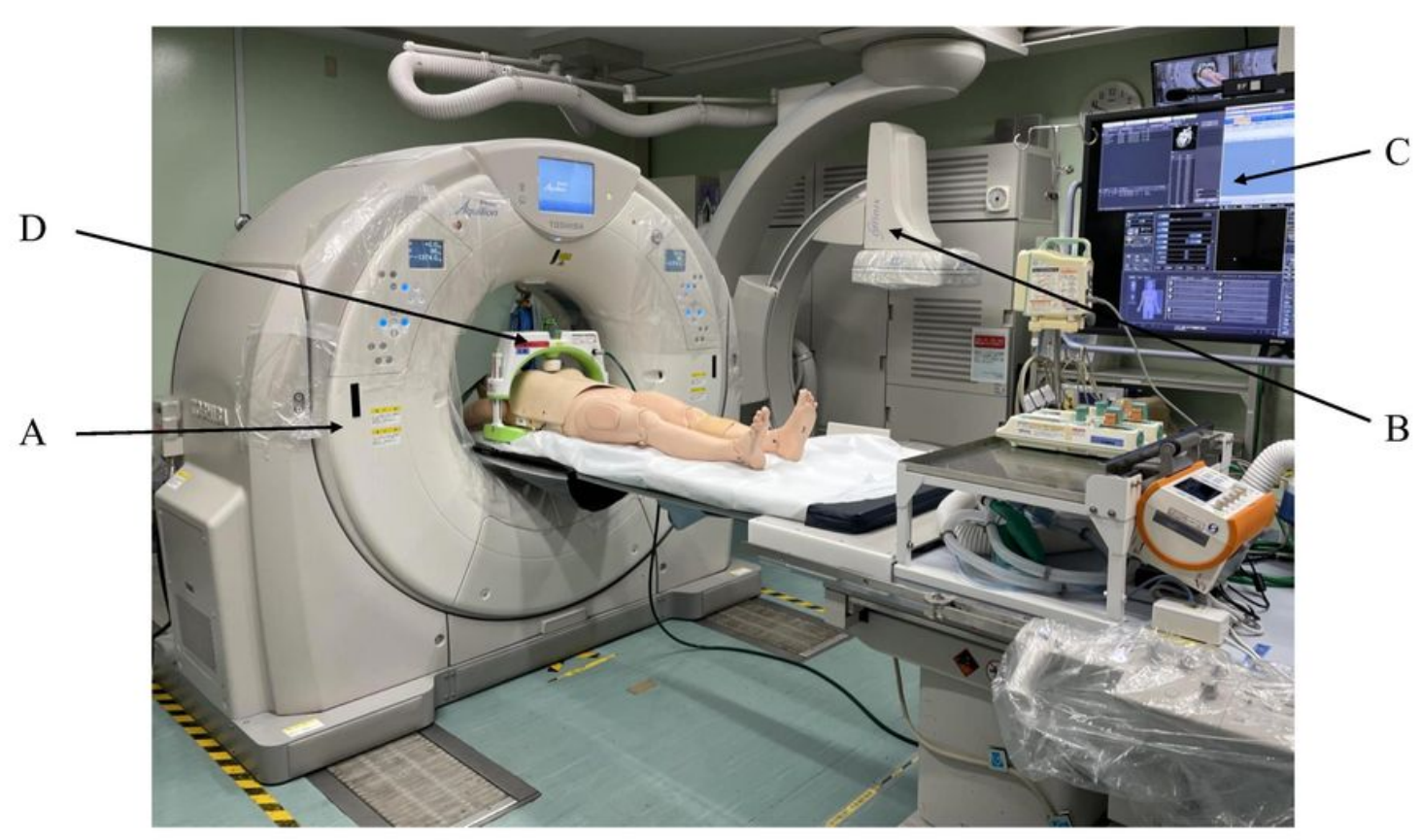

Figure 1

This photograph shows our hybrid ER and the scene while performing CT under mechanical CPR.

(A) sliding gantry computer tomography scanner, (B) movable C-arm, (C) monitoring screen, (D) automatic chest compression device

ER, Emergency room CT, Computed tomography; CPR, Cardiopulmonary resuscitation 


\section{Figure 2.}

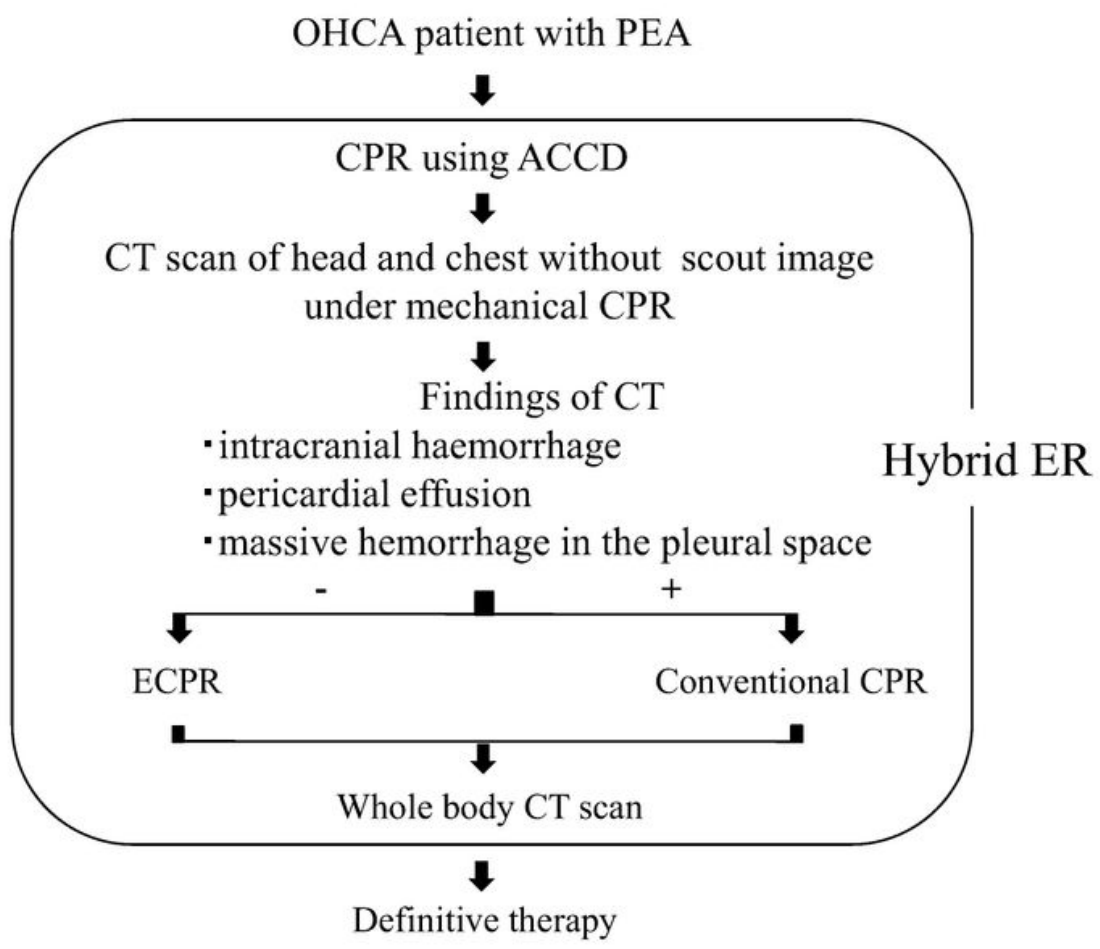

\section{Figure 2}

The schema of our protocol in selecting ECPR candidates with PEA.

PEA, Pulseless electrical activity; CPR, Cardiopulmonary resuscitation; ACCD, Automatic chest compression device; CT, Computed tomography; ECPR, extracorporeal cardiopulmonary resuscitation 


\section{Figure 3.}

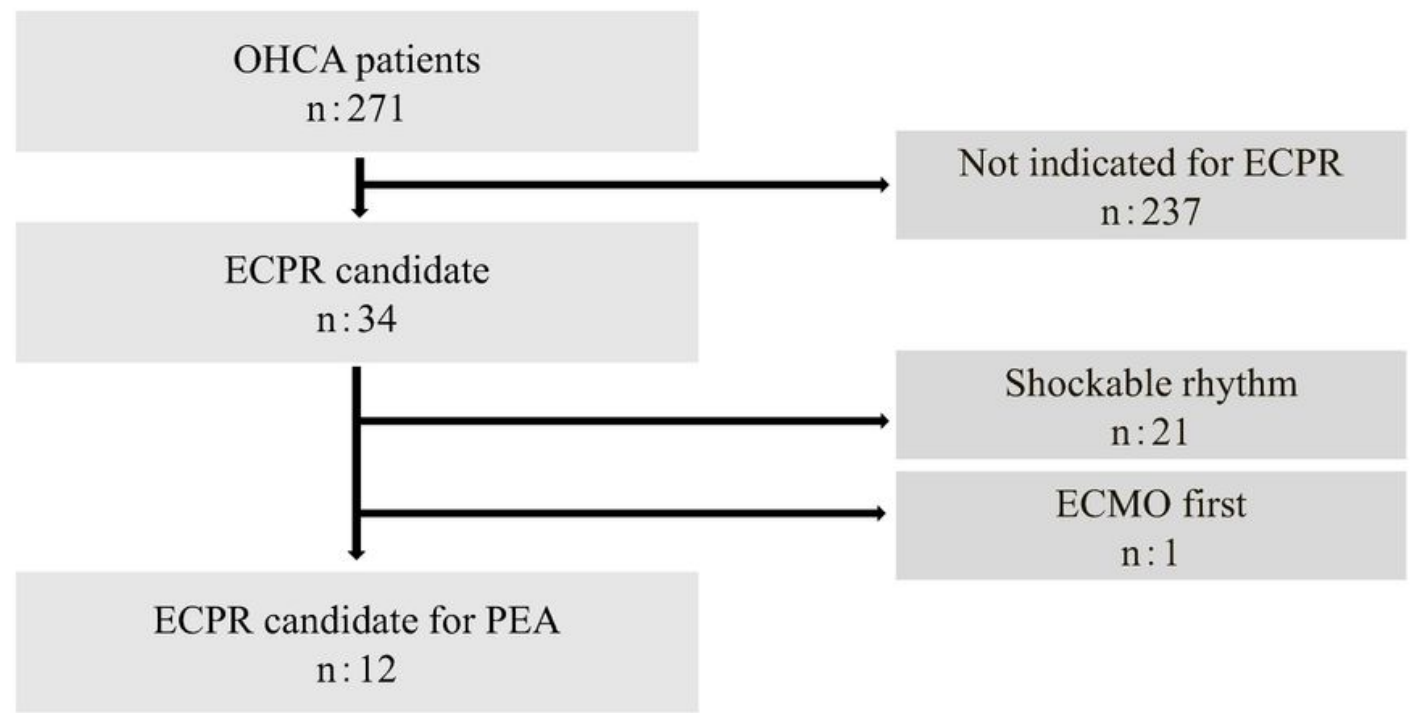

\section{Figure 3}

Flowchart of study patients.

OHCA, Out-of-hospital cardiac arrest; ECPR, extracorporeal cardiopulmonary resuscitation; ECMO, extracorporeal membrane oxygenation 


\section{Figure 4.}
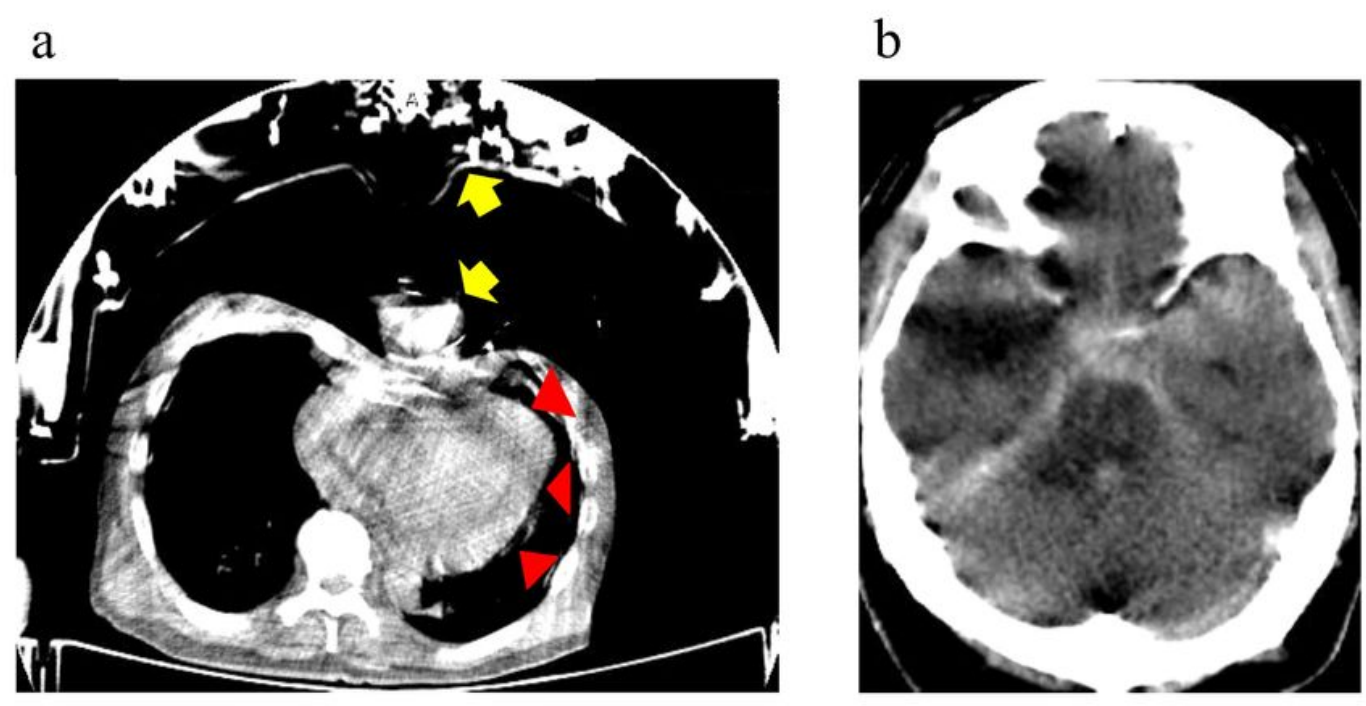

Figure 4

CT scan under mechanical CPR

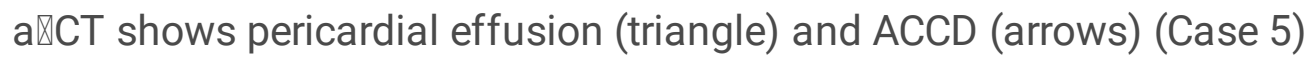

$\mathrm{b} \otimes C T$ shows subarachnoid haemorrhage (Case 7)

CT: Computed tomography, CPR: Cardiopulmonary resuscitation, ACCD: Automatic chest compression device

\section{Supplementary Files}

This is a list of supplementary files associated with this preprint. Click to download.

- HEMCstrategyTableSJTREM.pdf 